\title{
MOLECULAR OUTFLOWS IN B335 AND B1
}

\author{
N. HIRANO ${ }^{1}$, O. KAMEYA ${ }^{2}$, T. KASUGA ${ }^{3}$, H. MIKAMI ${ }^{4}$, S. SAITO $^{5}$, \\ T. UMEMOTO ${ }^{6}$, AND S. YAMAMOTO ${ }^{4}$ \\ ${ }^{1}$ Laboratory of Astronomy and Geophysics, Hitotsubashi University \\ 2Mizusawa Astrogeodynamics Observatory,National Astronomical Observatory \\ ${ }^{3}$ Department of Instrument and Control Engineering, College of Engineering, \\ Hosei University \\ ${ }^{4}$ Department of Astrophysics, Nagoya University \\ Institute for Molecular Science \\ 6Nobeyama Radio Observatory, National Astronomical Observatory
}

We present the aperture synthesis observations of the $\mathrm{CO}$ molecular outflows associated with the low-mass young stellar objects embedded in B335 and B1. We used the Nobeyama Millimeter Array and obtained the angular resolutions of 8.1" x 5.0" for B335 and $6.5^{\prime \prime} \times 4.4^{\prime \prime}$ for B1.

\section{1) $B 335$}

B335 is well studied as a typical bipolar outflow source. The high-resolution singledish maps of $\mathrm{CO}(\mathrm{J}=1-0)$ (Hirano et al. 1988; Moriarty-Schieven \& Snell 1989) reveal that the outflow is of biconical shape and is centered at the cold IRAS source, 19347+0727. B335 is the most suitable object to study the detailed structure of the outflow in the close vicinity of its driving source, because the outflow axis has a small inclination angle $\left(\sim 10^{\circ}\right)$ to the plane of the sky.

In Figure 1 we present the distribution of the blueshifted emission $\left(V_{L S R}=7.84 \mathrm{~km}\right.$ $\mathrm{s}^{-1}$; solid contours), the redshifted emission (VLSR $=9.46 \mathrm{~km} \mathrm{~s}^{-1}$; dashed contours), and the $2.6 \mathrm{~mm}$ continuum emission (gray scale). The blueshifted and redshifted $\mathrm{CO}$ emission shows distinct biconical structure. Two prominent peaks are separated by 10 " (=2400 AU) in blueshifted component and 15" (= $3600 \mathrm{AU}$ ) in redshifted one. The north-south extent of the outflow at its center is less than $8^{\prime \prime}(=2000 \mathrm{AU})$, indicating that the outflow is focused within $\sim 1000 \mathrm{AU}$ of its origin. The $2.6 \mathrm{~mm}$ continuum peak whose flux is $\sim 80 \mathrm{mJy}$ is located within 4 " of the outflow center. The $2.6 \mathrm{~mm}$ continuum flux combined with the previous far-infrared and submillimeter measurements (Keene et al. 1983; Gee et al. 1985; Chandler et al. 1990; IRAS Point Source Catalog, Version 2, 1988) suggest that the very dense $\left(>108 \mathrm{~cm}^{-3}\right)$ and massive $\left(1.7-2.9 \mathrm{M}_{\odot}\right)$ core is surrounding the young stellar object; such high density material may be responsible for collimating the outflow. In the vicinity of the origin, the flow velocity shows a sudden increase away from the center (see Figure 3 of Hirano et al. 1992). A simple dynamical model of the expanding "snowplowing" shell can explain the velocity field of B335.

\section{2) $B 1$}

B1 contains a low-luminosity IRAS source $03301+3057$, toward which high-velocity CO emission was detected (Nakayama 1988; Bachiller et al. 1990). The high-velocity emission is confined within $40^{\prime \prime}(0.07 \mathrm{pc}$ at the distance of $350 \mathrm{pc})$ of the IRAS source and is dominated by the blueshifted material. The dynamical timescale of the outflow is estimated to be less than $104 \mathrm{yr}$ (Nakayama 1988) or less than $103 \mathrm{yr}$ (Bachiller et al. 1990), indicating that the outflow is in the early stage of its growth. Furthermore, detection of the 
thermal emission lines of SiO (Bachiller et al. 1990; Martin-Pintado, Bachiller, \& Fuente 1992; Yamamoto et al. 1992) suggests the existence of some violent activities comparable to those of massive star formation.

The $\mathrm{CO}$ emission is detected in the velocity range from -1 to $5.5 \mathrm{~km} \mathrm{~s}^{-1}$, which is blueshifted relative to the ambient cloud velocity of $6.3 \mathrm{~km} \mathrm{~s}^{-1}$. No significant emission is seen in the velocity larger than $6.3 \mathrm{~km} \mathrm{~s}-1$. This indicates that the outflow in B1 is asymmetric in the size of a few thousand $\mathrm{AU}$ and is dominated by blueshifted material. Figure 2 shows the total integrated intensity map of $\mathrm{CO}(\mathrm{J}=1-0)$, superposed on the gray scale map of SiO (J=2-1: Yamamoto et al. 1992). The blueshifted gas shows distinct ring-like structure centered at IRAS $03301+3057$. The size of the CO ring is $25^{\prime \prime}(=8700 \mathrm{AU}) \times 14^{\prime \prime}(=5000$ $A U)$. The central hole of $C O(J=1-0)$ emission well coincide with the IRAS position; this suggests that the observed structure is a nearly pole-on view of the molecular shell swept up by the wind from IRAS $03301+3057$. The CO emission ring lies between two clumps of $\mathrm{SiO}$; the distribution of the $\mathrm{CO}$ emission appears to be anti-correlate with that of the SiO. The $\mathrm{H} 13 \mathrm{CO}+$ (Yamamoto et al. 1992) and $\mathrm{NH}_{3}$ (Bachiller et al. 1990) results suggest that the dense gas exists at the southeast of the IRAS source. The morphological relation among the $\mathrm{CO}$ outflow, $\mathrm{SiO}$ clumps, and dense gas implies that the outflow interacts with the dense gas and causes the strong shock which produce the $\mathrm{SiO}$.

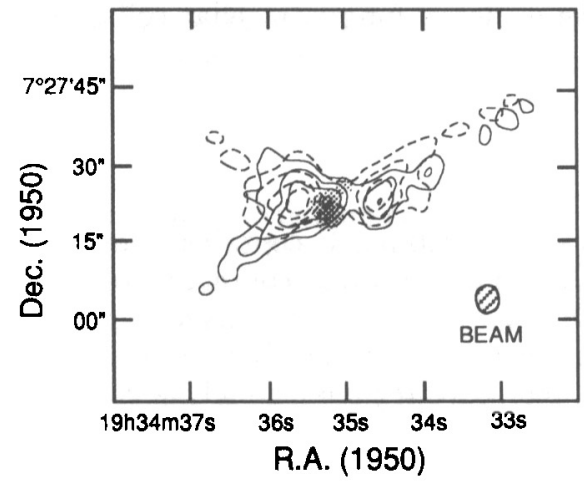

Fig. 1 Contour plots of the blueshifted emission (solid), redshifted emission (dashed), and the $2.6 \mathrm{~mm}$ continuum emission (gray scale) of B335.

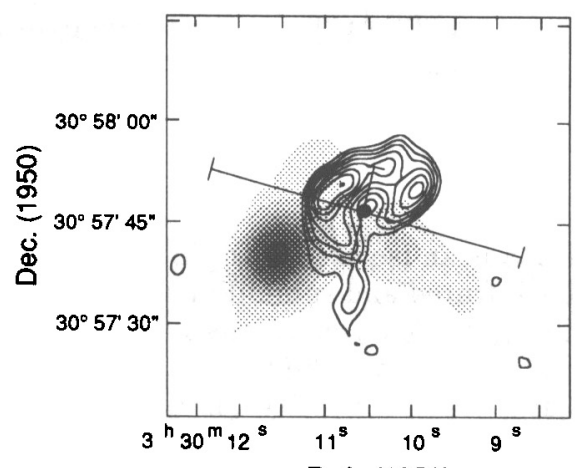

R. A. (1950)

Fig. 2 Integrated intensity map of the $\mathrm{CO}$ emission of B 1, superposed on the gray scale map of SiO (Yamamoto et al. 1992). The cross mark indicates the IRAS position.

\section{REFERENCES}

Bachiller, R., Menten, K.M., \& del Rio-Alvarez, S. 1990, A\&A, 236, 461

Chandler, C.J. et al. 1990, MNRAS, 243, 330

Gee, G. et al. 1985, MNRAS, 215, 15

Hirano, N., Kameya, O., Nakayama, M., \& Takakubo, K. 1988, ApJ, 327, L69

Hirano, N., Kameya, O., Kasuga, T., \& Umemoto, T. 1922, ApJ, 390, L85

Keene, J. et al. 1983, ApJ, 274, L43

Martin-Pintado, J., Bachiller, R., \& Fuente, A. 1992, A\&A, 254, 315

Moriarty-Schieven, G.H., \& Snell, R.L. 1989, ApJ, 338, 952

Nakayama, M. 1988, M.S. Thesis, Tohoku University

Yamamoto, S. et al. 1992, PASJ, 44, 459 